\title{
Chapter 90 \\ Report on the Global Environment \\ Competitiveness of Mali
}

Mali is located in West Africa. It covers 1,220,190 square kilometres and borders Algeria to the north, Niger to the east, Burkina Faso and to the south, Guinea to the south-west, and Senegal and Mauritania to the west. Mali's climate ranges from tropical in the south to arid in the north. Most of the country receives negligible rainfall and droughts are frequent. It had a population of 15.8 million and domestic production the gross (GDP) of USD 10.5 billion in 2011. Through the evaluation of global environment competitiveness, we can know that the environment competitiveness index of Mali ranks at 130 among 133 countries.

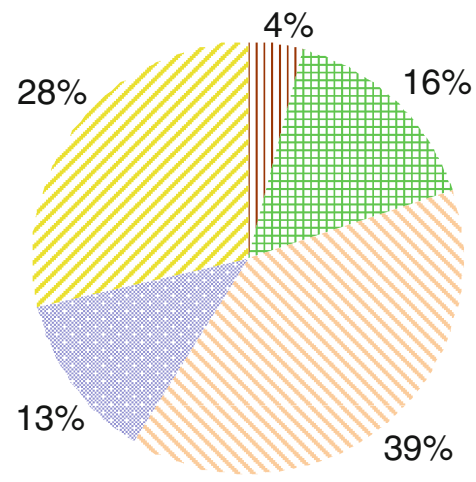

III Resource Environment Competitiveness

Ecological Environment Competitiveness

Environment Carrying Competitiveness

Environment Management Competitiveness

Environment Harmony Fig. 90.1 Contribution of Competitiveness sub-index of GEC 


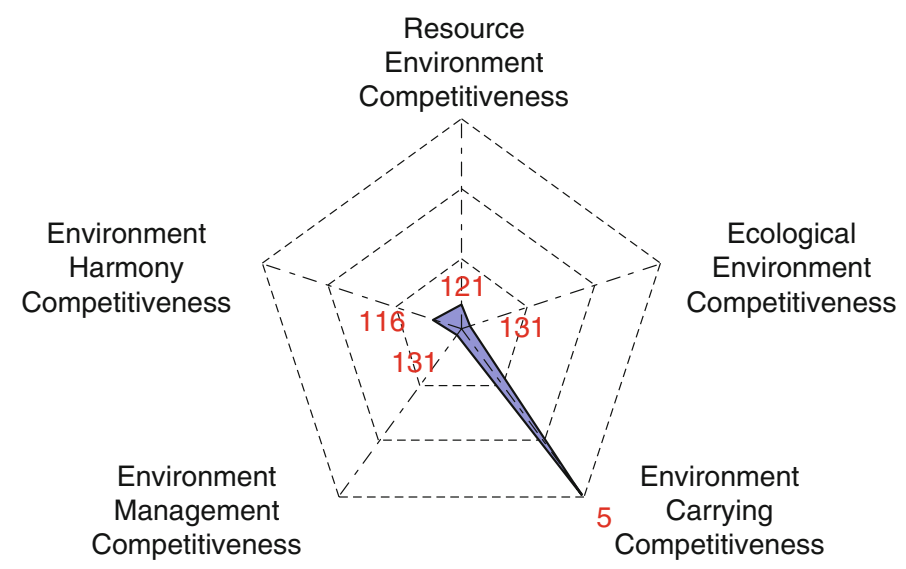

Fig. 90.2 Rank of sub-index of GEC

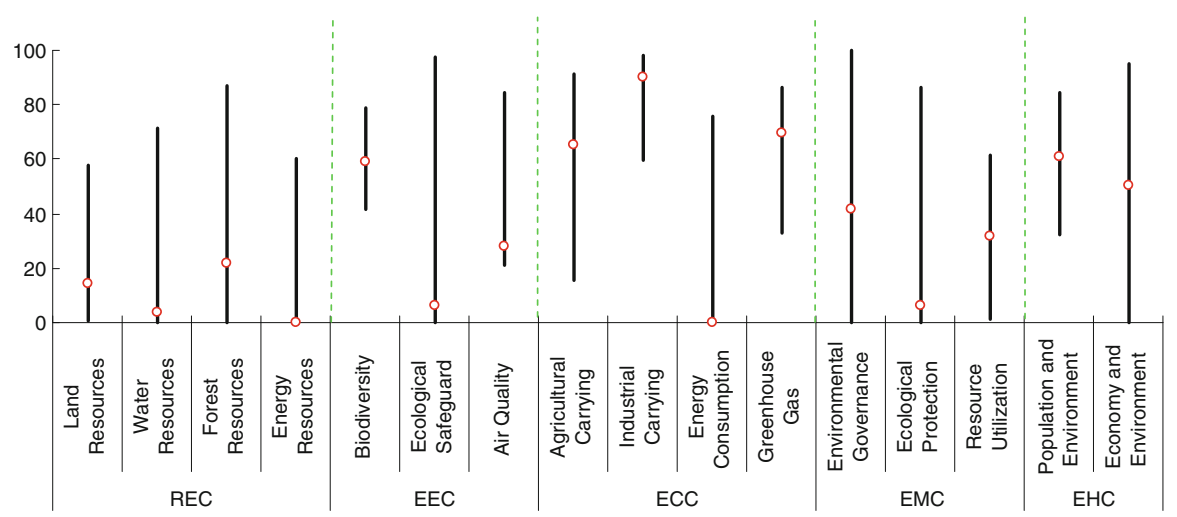

Fig. 90.3 Score and rank of the pillars of GEC

Table 90.1 Score and rank of all indicators of GEC

\begin{tabular}{|c|c|c|c|c|c|}
\hline Indicators & Score & Rank & Indicators & Score & Rank \\
\hline 1 Resource Environment & 8.36 & 121 & 1.3 Forest Resources & 21.63 & 88 \\
\hline Competitiveness & & & \multirow{2}{*}{$\begin{array}{l}\text { Growing stock in forest } \\
\text { and other wooded land }\end{array}$} & \multirow{2}{*}{50.75} & \multirow{2}{*}{79} \\
\hline 1.1 Land Resources & 14.44 & 55 & & & \\
\hline Land area per capita & 13.86 & 16 & \multirow{2}{*}{$\begin{array}{c}\text { Proportion of land area } \\
\text { covered by forest }\end{array}$} & \multirow[t]{2}{*}{11.91} & \multirow[t]{2}{*}{98} \\
\hline Percentage of arable land & 9.39 & 102 & & & \\
\hline to total land area & & & Forest area per capita & 5.46 & 31 \\
\hline Arable land per capita & 20.28 & 21 & 1.4 Energy Resources & 0.00 & 125 \\
\hline 1.2 Water Resources & 3.83 & 112 & Fossil energy & 0.00 & 64 \\
\hline Surface water & 0.80 & 102 & Energy production & N/A & N/A \\
\hline Annual precipitation & 9.72 & 110 & \multirow{3}{*}{$\begin{array}{l}\text { Proportion of combustible } \\
\text { renewables and waste } \\
\text { to total energy } \\
\text { consumption }\end{array}$} & \multirow[t]{3}{*}{ N/A } & \multirow[t]{3}{*}{ N/A } \\
\hline Groundwater & 2.32 & 101 & & & \\
\hline $\begin{array}{l}\text { Total internal renewable } \\
\text { water resources }\end{array}$ & 2.48 & 106 & & & \\
\hline
\end{tabular}


Table 90.1 (continued)

\begin{tabular}{|c|c|c|c|c|c|}
\hline Indicators & Score & Rank & Indicators & Score & Rank \\
\hline $\begin{array}{l}\text { Net energy imports of the } \\
\text { energy consumption }\end{array}$ & N/A & N/A & $\begin{array}{l}\text { Ratio of clean energy } \\
\text { consumption }\end{array}$ & 0.00 & 116 \\
\hline $\begin{array}{l}2 \text { Ecological Environment } \\
\text { Competitiveness }\end{array}$ & 30.68 & 131 & $\begin{array}{l}\text { Elasticity of energy } \\
\text { consumption }\end{array}$ & N/A & N/A \\
\hline 2.1 Biodiversity & 58.93 & 34 & \multirow{2}{*}{$\begin{array}{l}\text { Elasticity of electric power } \\
\text { consumption }\end{array}$} & \multirow[t]{2}{*}{ N/A } & \multirow[t]{2}{*}{ N/A } \\
\hline Threatened fish species & 98.58 & 9 & & & \\
\hline $\begin{array}{l}\text { Threatened mammal } \\
\text { species }\end{array}$ & 93.48 & 72 & \multirow{2}{*}{$\begin{array}{l}\text { Growth rate of } \mathrm{CO}_{2} \\
\text { emissions }\end{array}$} & \multirow{2}{*}{$\begin{array}{l}69.45 \\
54.18\end{array}$} & $\begin{array}{l}23 \\
55\end{array}$ \\
\hline Threatened plant species & 99.59 & 54 & & & 55 \\
\hline $\begin{array}{l}\text { GEF benefits index for } \\
\text { biodiversity }\end{array}$ & 1.50 & 75 & $\begin{array}{l}\text { Growth rate of Methane } \\
\text { emissions }\end{array}$ & N/A & N/A \\
\hline 2.2 Ecological Safeguard & 6.25 & 109 & \multirow{2}{*}{$\begin{array}{l}\mathrm{CO}_{2} \text { emissions per unit } \\
\text { of land area }\end{array}$} & \multirow[t]{2}{*}{100.00} & \multirow[t]{2}{*}{1} \\
\hline Terrestrial protected areas & 6.25 & 111 & & & \\
\hline Marine protected areas & N/A & N/A & \multirow{2}{*}{$\begin{array}{l}\mathrm{CO}_{2} \text { emissions per unit } \\
\text { of energy consumption }\end{array}$} & \multirow[t]{2}{*}{ N/A } & \multirow[t]{2}{*}{ N/A } \\
\hline 2.3 Air Quality & 27.82 & 130 & & & \\
\hline Inhalable particles (PM10) & 18.98 & 130 & \multirow{2}{*}{$\begin{array}{l}\text { Competitiveness } \\
\text { Comironment }\end{array}$} & \multirow[t]{2}{*}{24.49} & \multirow{2}{*}{131} \\
\hline Particulate matter (PM2.5) & 67.95 & 113 & & & \\
\hline $\begin{array}{l}\text { Index of indoor air } \\
\text { pollution }\end{array}$ & 0.30 & 126 & $\begin{array}{l}\text { 4.1 Environmental } \\
\text { Governance }\end{array}$ & 41.40 & 127 \\
\hline Nitrogen oxides emission & 68.97 & 4 & $\begin{array}{l}\text { Agricultural chemicals } \\
\text { regulation }\end{array}$ & 0.00 & 86 \\
\hline Sulfur dioxide emission & N/A & N/A & \multirow{3}{*}{$\begin{array}{l}\text { Percentage of the rural } \\
\text { population with access } \\
\text { to an improved water } \\
\text { source }\end{array}$} & \multirow{3}{*}{51.00} & \multirow{3}{*}{107} \\
\hline $\begin{array}{l}3 \text { Environment Carrying } \\
\text { Competitiveness }\end{array}$ & 75.67 & 5 & & & \\
\hline 3.1 Agricultural Carrying & 65.14 & 80 & & & \\
\hline $\begin{array}{l}\text { Cereal yield per unit } \\
\text { of arable land }\end{array}$ & 14.02 & 101 & \multirow{2}{*}{$\begin{array}{l}\text { Percentage of the urban } \\
\text { population with access } \\
\text { to an improved water } \\
\text { source }\end{array}$} & \multirow[t]{2}{*}{87.00} & \multirow[t]{2}{*}{114} \\
\hline $\begin{array}{l}\text { Fertilizer consumption per } \\
\quad \text { unit of arable land }\end{array}$ & 99.75 & 12 & & & \\
\hline Annual freshwater & 98.69 & 67 & 4.2 Ecological Protection & 6.52 & 126 \\
\hline $\begin{array}{l}\text { withdrawals for } \\
\text { agriculture per unit } \\
\text { of arable land }\end{array}$ & & & $\begin{array}{l}\text { Area of plantation and } \\
\text { afforestation }\end{array}$ & 0.69 & 47 \\
\hline 3.2 Industrial Carrying & 90.36 & 80 & Biome protect & 14.30 & 110 \\
\hline $\begin{array}{l}\text { Net exports as a percentage } \\
\text { of GDP }\end{array}$ & 84.19 & 51 & $\begin{array}{l}\text { Overfishing of fishing } \\
\text { resources }\end{array}$ & N/A & N/A \\
\hline $\begin{array}{l}\text { Electric power } \\
\text { consumption per unit of } \\
\text { value added of industry }\end{array}$ & N/A & N/A & $\begin{array}{l}\text { 4.3 Resource Utilization } \\
\text { Utilization rate of water } \\
\text { resources }\end{array}$ & $\begin{array}{r}31.54 \\
0.26\end{array}$ & $\begin{array}{l}87 \\
69\end{array}$ \\
\hline $\begin{array}{l}\mathrm{SO}_{2} \text { emissions per unit of } \\
\text { value added of industry }\end{array}$ & N/A & N/A & \multirow{2}{*}{$\begin{array}{l}\text { Percentage of total internal } \\
\text { renewable water } \\
\text { resources to total water } \\
\text { resources }\end{array}$} & \multirow[t]{2}{*}{54.55} & \multirow[t]{2}{*}{82} \\
\hline Annual freshwater & 96.53 & 78 & & & \\
\hline $\begin{array}{l}\text { industry per value } \\
\text { added of industry }\end{array}$ & & & $\begin{array}{l}\text { Percentage of agricultural } \\
\text { land to total land area }\end{array}$ & 39.81 & 91 \\
\hline 3.3 Energy Consumption & N/A & N/A & \multirow{2}{*}{$\begin{array}{l}\text { Percentage of fossil fuel } \\
\text { energy consumption } \\
\text { to total energy } \\
\text { consumption }\end{array}$} & \multirow[t]{2}{*}{ N/A } & \multirow[t]{2}{*}{ N/A } \\
\hline $\begin{array}{l}\text { Energy consumption per } \\
\text { unit of land area }\end{array}$ & N/A & N/A & & & \\
\hline
\end{tabular}


Table 90.1 (continued)

\begin{tabular}{|c|c|c|c|c|c|}
\hline Indicators & Score & Rank & Indicators & Score & Rank \\
\hline $\begin{array}{l}5 \text { Environment Harmony } \\
\text { Competitiveness }\end{array}$ & 55.42 & 116 & $\begin{array}{l}\mathrm{CO}_{2} \text { emissions (metric tons } \\
\text { per capita) }\end{array}$ & 100.00 & 1 \\
\hline $\begin{array}{l}\text { 5.1 Population and } \\
\text { Environment }\end{array}$ & 60.84 & 115 & $\begin{array}{l}\text { Energy consumption } \\
\text { per capita }\end{array}$ & N/A & N/A \\
\hline $\begin{array}{l}\text { Improved sanitation } \\
\text { facilities }\end{array}$ & 36.00 & 106 & $\begin{array}{l}\text { 5.2 Economy and } \\
\text { Environment }\end{array}$ & 50.00 & 112 \\
\hline $\begin{array}{l}\text { (\% of population } \\
\text { with access) }\end{array}$ & & & $\begin{array}{l}\text { Land resource utilization } \\
\text { efficiency }\end{array}$ & 0.00 & 130 \\
\hline $\begin{array}{l}\text { Motor vehicles } \\
\quad \text { (per 1,000 people) }\end{array}$ & 98.52 & 16 & $\begin{array}{l}\text { Sulfur dioxide emissions } \\
\text { per unit of GDP }\end{array}$ & N/A & N/A \\
\hline $\begin{array}{l}\text { Renewable internal } \\
\text { freshwater resources }\end{array}$ & 4.58 & 57 & $\begin{array}{l}\text { Carbon dioxide emissions } \\
\text { per unit of GDP }\end{array}$ & 100.00 & 1 \\
\hline $\begin{array}{l}\text { per capita } \\
\mathrm{SO}_{2} \text { emissions (metric tons } \\
\text { per capita) }\end{array}$ & N/A & N/A & $\begin{array}{l}\text { Energy consumption } \\
\text { per unit of GDP }\end{array}$ & N/A & N/A \\
\hline
\end{tabular}

Table 90.2 Rank distribution of the individual indicators of GEC

\begin{tabular}{|c|c|c|c|c|c|c|}
\hline Sub-index & $\begin{array}{l}\text { Number } \\
\text { of the } \\
\text { individual } \\
\text { indicators }\end{array}$ & $\begin{array}{l}\text { Rank } \\
1-10\end{array}$ & $\begin{array}{l}\text { Rank } \\
11-30\end{array}$ & $\begin{array}{l}\text { Rank } \\
31-60\end{array}$ & $\begin{array}{l}\text { Rank } \\
61-100\end{array}$ & $\begin{array}{l}\text { Rank } \\
101-133\end{array}$ \\
\hline $\begin{array}{l}\text { Resource Environment } \\
\text { Competitiveness }\end{array}$ & 14 & 0 & 1 & 1 & 4 & 6 \\
\hline $\begin{array}{l}\text { Ecological Environment } \\
\text { Competitiveness }\end{array}$ & 11 & 2 & 0 & 2 & 1 & 6 \\
\hline $\begin{array}{l}\text { Environment Carrying } \\
\text { Competitiveness }\end{array}$ & 15 & 1 & 2 & 2 & 2 & 2 \\
\hline $\begin{array}{l}\text { Environment Management } \\
\text { Competitiveness }\end{array}$ & 10 & 0 & 0 & 1 & 5 & 4 \\
\hline $\begin{array}{l}\text { Environment Harmony } \\
\text { Competitiveness }\end{array}$ & 10 & 2 & 1 & 1 & 0 & 4 \\
\hline Total & 60 & 5 & 4 & 7 & 12 & 22 \\
\hline
\end{tabular}

Open Access This chapter is distributed under the terms of the Creative Commons Attribution Noncommercial License, which permits any noncommercial use, distribution, and reproduction in any medium, provided the original author(s) and source are credited. 